\title{
A Full-length Quench Antenna Array for MQXFA Production Series Quadrupole Magnet Testing
}

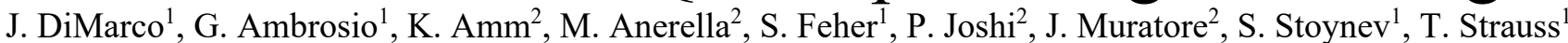

\author{
1. Fermilab, Batavia, IL, United States. 2. Brookhaven National Lab, Upton, NY, United States.
}

\begin{abstract}
The MQXFA production series quadrupoles being built for the Hi-Lumi (HL) LHC upgrade by the US Accelerator Upgrade Project (US-HL-LHC AUP) will have very limited voltage instrumentation for characterizing quench events that occur during magnet training and performance validation testing. In order to understand the origin of the quenches, and whether they have some implication for ongoing magnet fabrication, a fulllength Quench Antenna Array (QAA) was built with axial resolution of $50 \mathrm{~mm}$ to be employed during cold testing in the anticryostat. The goal is to have fine-resolution, full-length coverage detection of quench events axially, as well as to have azimuthal resolution on the order of the cable width (about 1 degree for the cross-section), in a device that can be used both for vertical and horizontal testing. To achieve this, a $5 \mathrm{~m}$ long QAA with 128 channels of high-speed data acquisition has been designed and fabricated. The array features full-length radially positioned antennas for azimuthal localization, and short, high-sensitivity, antennas for axial detection. This paper discusses the design, construction and analysis of the MQXFA QAA, and first results from its use during quench testing in the production magnets.
\end{abstract}

Index Terms - Magnetic field measurement, quench antenna, quench detection, quench characterization, circuit board probe

\section{INTRODUCTION}

A s part of the US-HiLumi Accelerator Upgrade Project (AUP), Fermilab, together with Brookhaven National Lab (BNL) and Lawrence Berkeley National Lab (LBNL) are fabricating $204.5 \mathrm{~m}$-long $\mathrm{Nb} 3 \mathrm{Sn}$ superconducting magnets to be used in the interaction regions of the LHC. These magnets are undergoing vertical test at Brookhaven to verify performance specifications. Afterwards they will be sent to Fermilab for incorporation, pair-wise as a cold-mass, into cryogenic vessels, where they will be tested once again horizontally in their final configuration. Since these are production magnets to be installed in the High-Luminosity LHC at CERN, a minimum of diagnostic voltage taps used to characterize quench events are included during assembly. Such diagnostics add risk and substantial time to fabrication and test schedules, and, if the production run is going well, in principle would not need to be employed. However, quenches are not uncommon as part of normal magnet training procedures, and information on the details of quench events could be used to understand something about the fabrication process and even

This work was supported by the U.S. Department of Energy, Office of Science, Office of High Energy Physics, through the US LHC Accelerator Upgrade Project (AUP).

Corresponding author J. DiMarco (e-mail: dimarco@fnal.gov). provide feed-back with which to improve it. With the twin goals of minimizing impact on magnet construction and yet capturing all quench information, a full-length quench antenna array has been fabricated at Fermilab. The QAA will be placed in the anti-cryostat used for magnetic measurements, and will provide characterization of quenches during the vertical cold magnet testing at BNL. The quench antenna design which achieves complete axial coverage with fine resolution $(\sim 50 \mathrm{~mm}$ in straight section and $25 \mathrm{~mm}$ resolution in the ends), no dead zones, and which also has azimuthal localization capabilities, is described in detail herein, along with presentation of first results.

\section{DESIGN}

The QAA has a $4.8 \mathrm{~m}$ active length and $5.2 \mathrm{~m}$ overall length, to provide full quench detection coverage for the $4.5 \mathrm{~m}$ long MQXFA magnet with $4.2 \mathrm{~m}$ straight section. Two types of induction-sensitive QA are employed in the array: 111 antennas along the axis (referred to as Z-antennas, or ZQA), for determining axial location, and 12 antennas distributed azimuthally (so-called theta-antennas, or TQA) to detect angular location within the cross-section. Both ZQA and TQA feature dipole-quadrupole bucking (detailed further in Section III) to reduce sensitivity to non-quench events.

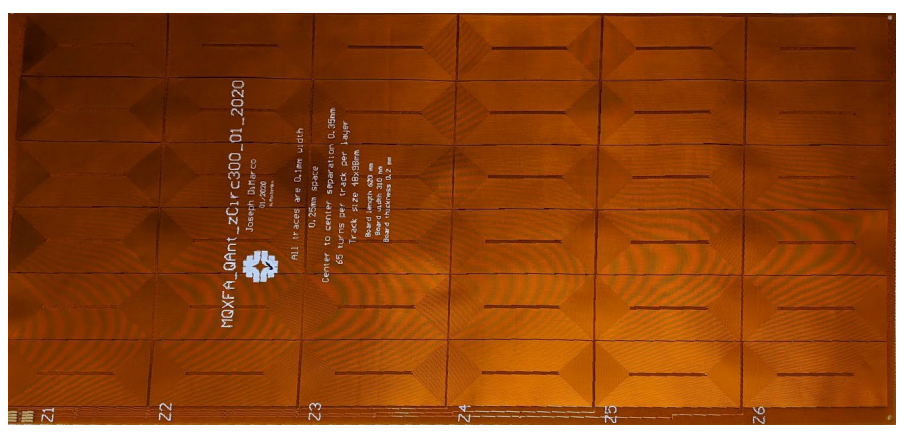

Fig.1. A Z-quench antenna panel with six $100 \mathrm{~mm} \times 300 \mathrm{~mm}$ 6-fold symmetric circuits. Labels $\mathrm{Z1}-\mathrm{Z6}$ are just visible at the bottom of the photo.

The ZQA are made with short axial extent so that they provide local detection of quenches with high resolution along the magnet length. The ZQA are composed of a 6-fold set of 'racetrack' windings, with each winding connected in series and alternating in chirality with its neighbor, to form a single circuit. These are shown going from top to bottom in the photo of Fig.1, which also shows 6 such independent circuits, end to end, manufactured on a single flexible Printed Circuit Board 'panel'. Each racetrack has 132 turns (66 per layer) and is 100 $\mathrm{mm}$ long and $50 \mathrm{~mm}$ wide. The six independent circuits on each panel give a total panel size of $600 \mathrm{~mm}$ x $300 \mathrm{~mm}$, and 
these are wrapped around the TQA assembly to form a cylinder with $\sim 95 \mathrm{~mm}$ diameter. The panel thickness is $0.2 \mathrm{~mm}$. The sensitivity of the racetrack windings to the quench event will depend on the angular position of the quench (e.g. close to the edge of a racetrack or near center), and since the alternating 6fold loop pattern will have regions in theta were the sensitivity is at or near zero, panels overlap by half the width and length of the loops to insure there are no gaps in sensitivity. A view of the panel overlapping is shown in Fig. 2. Given the overlap throughout, and double density in the ends for finer resolution, a total of 20 of the ZQA panels were needed to cover the $4.8 \mathrm{~m}$ $\mathrm{QA}$, extending from $\mathrm{Z}=-2300 \mathrm{~mm}$ to $Z=+2500 \mathrm{~mm}$ as positioned in the MQXFA04 magnet.

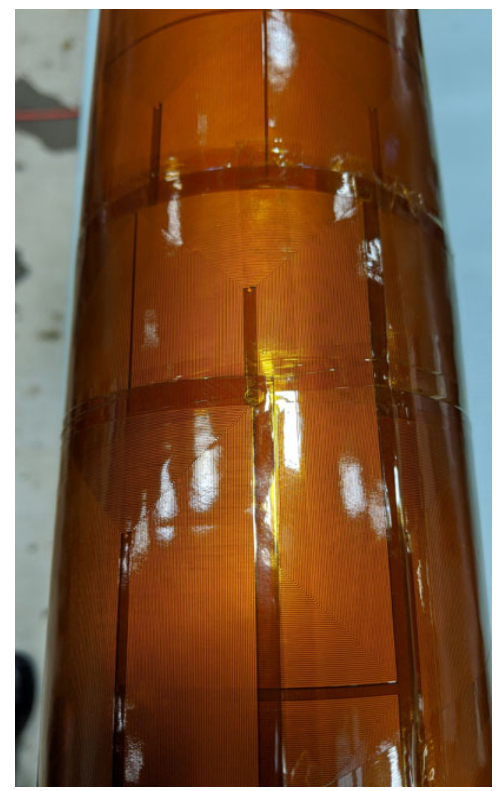

Fig.2. Close-up of the overlapping ZQA as mounted over the TQA.

ZQA resolution is $50 \mathrm{~mm}$ when the quench angle is such that the signal appears on both overlapped panels, or, if the quench is near one of the minima, resolution of $100 \mathrm{~mm}$. A length of $0.5 \mathrm{~m}$ at each end of the QA has higher density of ZQA with resolution $25 \mathrm{~mm}$ nominal $(50 \mathrm{~mm}$ worst-case). The fine resolution extends to fully cover both ends of the magnet if the QA center is placed within $0.15 \mathrm{~m}$ of magnet center.

The TQA are distributed in theta every 30 degrees in the QA interior, underneath the circumferentially wrapped ZQA. These extend the full $4.8 \mathrm{~m}$ active length of the probe in order to limit the total number of channels. With this length, making rigid or flexible printed circuits with high density becomes very costly. Instead, we made the full-length TQA out of ribbon cable with small circuit boards at each end to create the DQ-bucked circuit from the $25 \mathrm{~mm}$-wide, 40 conductor cable (Fig. 3). Insulation Displacement Connectors are used to minimize soldering. The 40 wires become 4 separate loops with 10 wires ( 5 turns) each, and are connected so as to buck dipole and quad fields. To compensate for the weak sensitivity of these few turns, the TQA channels are outfitted with 1000x gain amplifiers (based on AD8221BR) included on the lead end PCB which makes-up the circuit of each TQA. These are located inside the QA, upstream of the external cables and readout electronics.

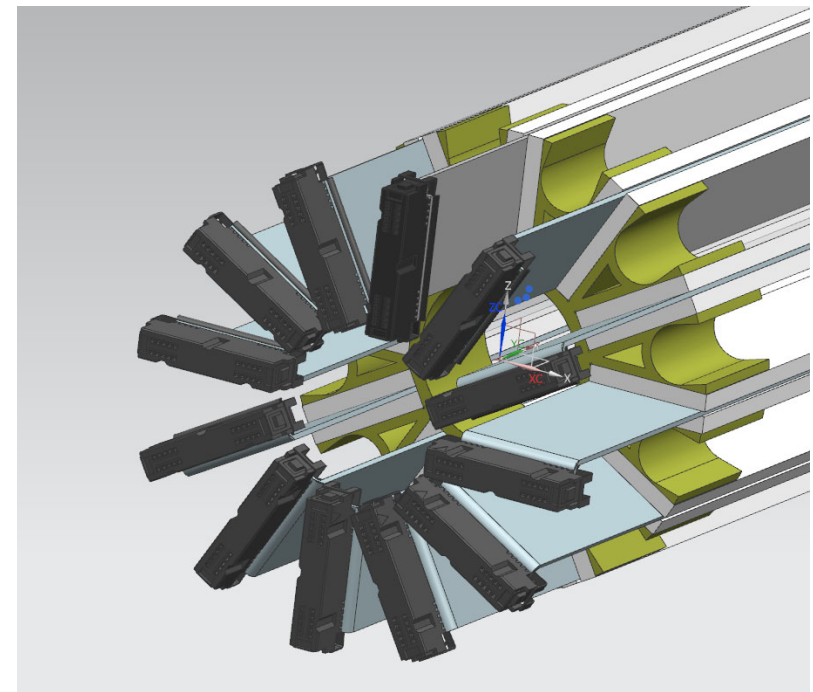

Fig.3. A model of the 3D-printed 'spiders' used to support the ribbon cables and connectors that comprise the Theta Quench Antennas.

The ribbon cables of the TQA are sandwiched in light-weight plastic stiffener and supported along the QA length every 150 $\mathrm{mm}$ in 3D-printed 'spiders'. The spider supports are aligned and glued onto the $33 \mathrm{~mm}$ OD central carbon-fiber core which bears the mechanical load of the assembly. The spiders also have areas which serve as conduit for the cabling of all channels. A view of the partially assembled QA, with TQA and amplifiers visible, is shown in Fig. 4.

The signals are brought out on individually-shielded twisted pair cables, with shielding extending all the way to the data acquisition system. Data acquisition consists of a rack with 128 channels of 16-bit resolution, simultaneously sampled, National Instruments ADCs running at $100 \mathrm{kHz}$ sampling frequency.

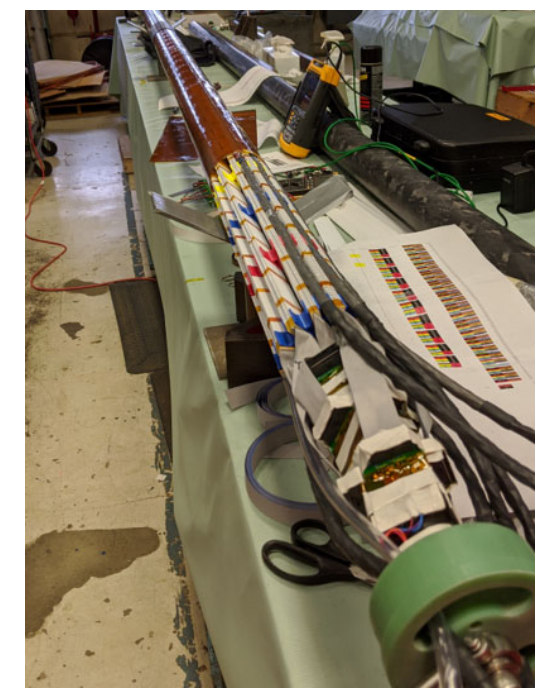

Fig.4. View of partially assembled QA. The TQA are visible in the foreground with their amplifier circuitry, and the ZQA in the background. Also visible is the carbon-fiber tube which slides over the assembly as an outer shell. 
The TQA, over-wrapped by the ZQA, are enclosed within a carbon-fiber tube with $98 \mathrm{~mm}$ OD and $95 \mathrm{~mm}$ ID, which comfortably can be placed within the $103 \mathrm{~mm}$ ID anti-cryostat used during cryogenic testing of MQXFA. A swivel hoist ring is placed at the top of the antenna for craning it into position vertically. The overall weight of the quench antenna is less than $20 \mathrm{~kg}$. A polypropylene tube also runs the full length of the antenna so that warm-up gas can be delivered to the bottom of the anti-cryostat.

\section{MEASUREMENT PRINCIPLE}

A quench developing in a superconducting magnet causes distortion in the local magnetic field because of current redistributions and/or motions in the cable. These small changes in field can be detected by a stationary wire pick-up loop of suitable sensitivity. Such a loop, or an array of such loops, is typically referred to as a 'quench antenna' (QA) [1]. Besides the quench induced changes, power supply ripple or fluctuations can cause substantial field changes which can obscure the QA signal, and so the effects of these field fluctuations need to be suppressed, or 'bucked' as much as possible (as for magnetic measurement probes [2]), by using a combination of loops that yield a net low sensitivity to quadrupole and dipole fields (DQ-bucked antennas).

For the Z-antennas, bucking is achieved by the sextupolesymmetric pattern of loops etched onto the flexible PCB panels. The bucking for the theta-antennas is accomplished with a series configuration of the 4 radial loops of the ribbon cable which suppresses the low order fields [2].

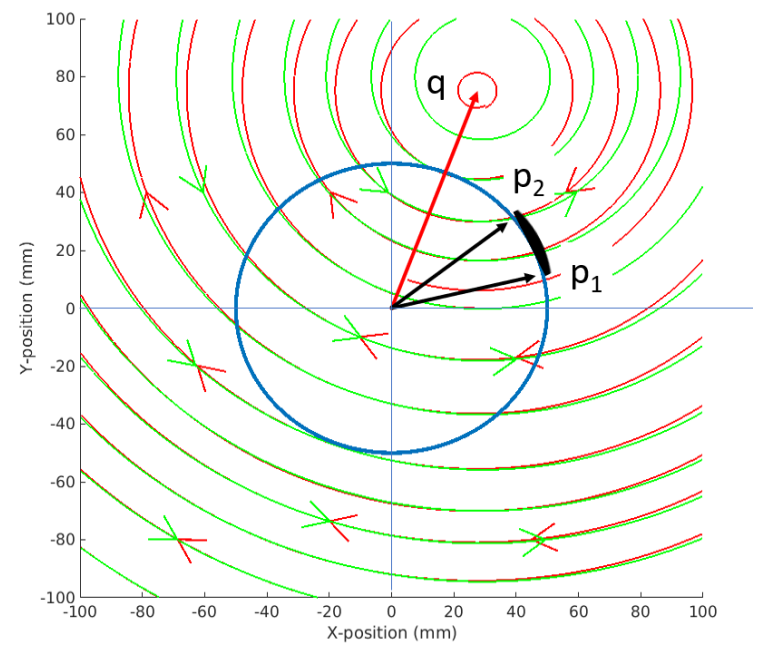

Fig.5. The field distortions from a quenching strand represented by a currentline doublet (positive line centered in red and negative, green). The circle centered on the origin represents the QAA structure in the magnet, having an inductive pick-up loop with vertices at locations designated by $\boldsymbol{p}_{1}$ and $\boldsymbol{p}_{2}$.

A quench event can be modelled as the appearance of a current line representing a new and shifted location of current within the magnet cable cross-section because of the development of a resistive zone, or more precisely, as that plus an oppositely signed current line that similarly represents the absence of current where it once flowed. This pair of current lines can be taken as causing the change in the field from the quench event, and, knowing the geometry of a wire loop in the vicinity, can be used to calculate the induced voltage observed in the loop (Fig. 5).

The field from a current line can be found from Ampere's law

$$
B(p)=\frac{\mu_{0} I}{2 \pi *(p-q)}
$$

where the $\boldsymbol{p}$ and $\boldsymbol{q}$ are complex positions of antenna wire locations and quench origin, respectively, in the coordinate cross-section of Fig. 5. To find the resultant voltage on the antenna, first we integrate the field with respect to $\boldsymbol{p}$ of a given winding to arrive at the complex potential

$$
W(p)=-\int B(p) d p=-\left(\frac{\mu_{0} I}{2 \pi}\right) \ln (p-q)
$$

which allows path-independent calculation of flux, i.e. using just the $\boldsymbol{p}_{j}$ vertex positions of a winding. Then, after multiplying by length, $L j$, of the antenna segment (or quenching length, whichever is shorter) to determine flux, Faraday's law is applied, so the voltage can be expressed as

$$
V=\sum_{j=1}^{N_{\text {wires }}}-1 * L_{j} * \frac{\mu_{0}}{2 \pi} * \frac{d I}{d t} * \ln \left(p_{j} *(-1)^{j}-q\right)
$$

Assuming radial current re-distribution distance of $1 \mathrm{~mm}$ (neighboring strand), and finding $\mathrm{dI} / \mathrm{dt}=\sim 2 \mathrm{e} 5 \mathrm{~A} / \mathrm{s}$ (from $\sim 500$ A current expected in a strand at high field with 1-2 milliseconds observed redistribution time), the voltage excursion expected can be found for various antenna designs. For the as-built ZQA, expected voltage of a quench event would be of order $40 \mathrm{mV}$, and for the TQA, about $100 \mu \mathrm{V}$.

\section{RESULTS}

First quench tests on MQXFA04 were conducted in August 2020 with the Quench Antenna installed in MQXFA04. The results of the quench testing are presented elsewhere at this conference [3]. A total of 8 quenches occurred in MQXFA04, 6 of which have recorded QA data. The quench is detected at " $t=0$ " and some number of "pre-quench" ADC samples are saved in the circular data buffer. For 5 out of 6 quenches, the pre-quench data extend back in time to $-0.1 \mathrm{~s}$. The last quench, Quench \#8, had pre-quench data going back to $-0.5 \mathrm{~s}$.

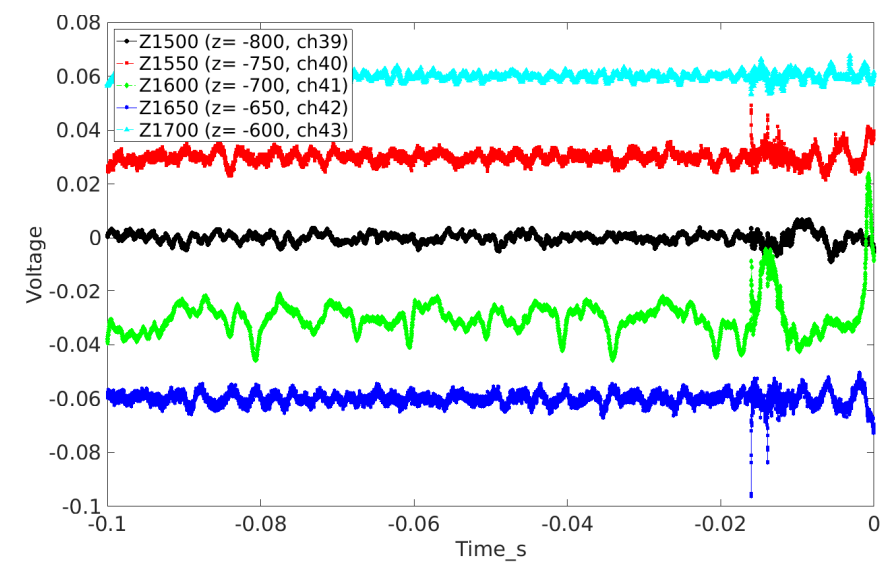

Fig.6. Raw ZQA Voltage signals, Quench \#1 (60Hz filtered). Note signals have been offset from one another for clarity. 
Sample raw data are shown in Fig. 6 for Quench \#1. Note that signal names represent their location relative to the start of the QA, thus "Z1500" is $1500 \mathrm{~mm}$ from the start of the active portion of the QA.

The data of Fig. 6 are unfiltered except for $60 \mathrm{~Hz}$ multiples. At first, more extensive filtering was applied to suppress the apparent noise and make the quenching segment stand out. However, in examining the signals at the end of the antenna, it was found that ZQA which extended furthest, past the end of the magnet, showed very little noise, though these had the longest cables of the QA. This suggested that the "noise" seen in the other axial antenna channels was actually induced voltage signal being picking up from current redistribution/vibrational motion during excitation.

The voltages can be integrated to show accumulated flux over time, which can be a more salient quench indicator (with the voltage offset being removed from the QA voltage before integration by averaging the first $50 \%$ of the signal). Representing this data in a surface plot (Fig. 7) allows for the first time a view of flux redistribution events along the entire magnet as a function of time. It is noted that there are several regions in $z$ that have high activity during more than one quench, and that the quenches observed in this magnet are located in these same areas.

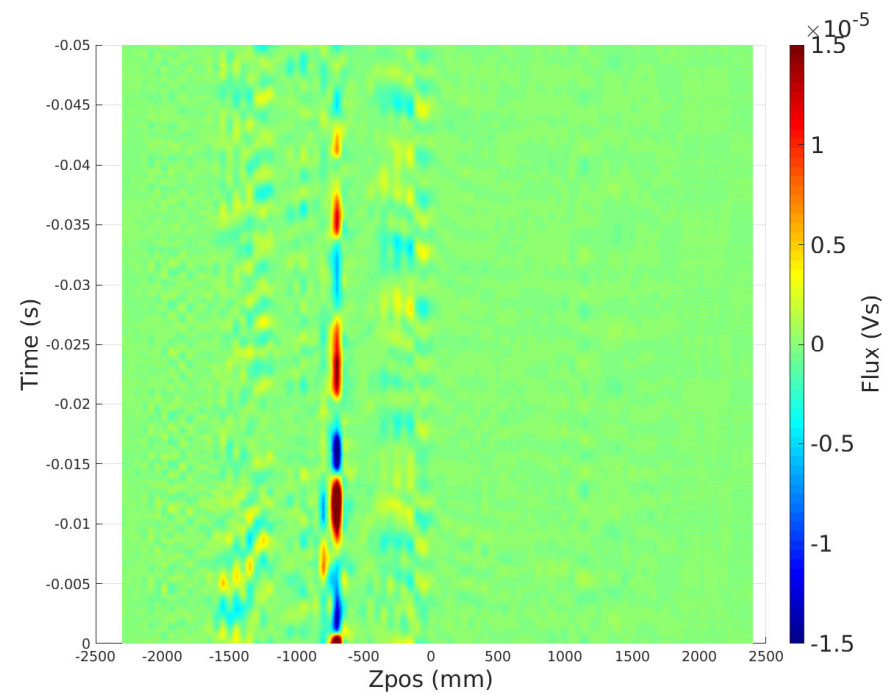

Fig.7. ZQA flux from Quench \#1 as a function of time and axial location. The quench event causes a large signal at $\sim \mathrm{t}=-0.015 \mathrm{~s}$. Pre-quench activity is seen in several areas, but predominantly in the quenching segment at $Z=-700 \mathrm{~mm}$.

Fig. 8 shows data from Quench 2, which has moved to center, where some activity was seen in Quench 1 . The region where Quench 1 occurred now has low activity, evidencing these measured voltages are not merely noisier signals. Note also that this quench seems of lower intensity than the first.

The voltage and flux ZQA results provide clear localization of activity for all the quenches of MQXFA04, as represented by the quenches included here, with localizations within the designed $25-50 \mathrm{~mm}$ resolution for all 6 recorded quenches. Some quenches have strong activity in more than one region as shown in Fig. 9 for Quench 3. The data also show evidence of quench propagation within the magnet (Fig. 10). An example of a quench in the magnet end is shown in Fig. 11.

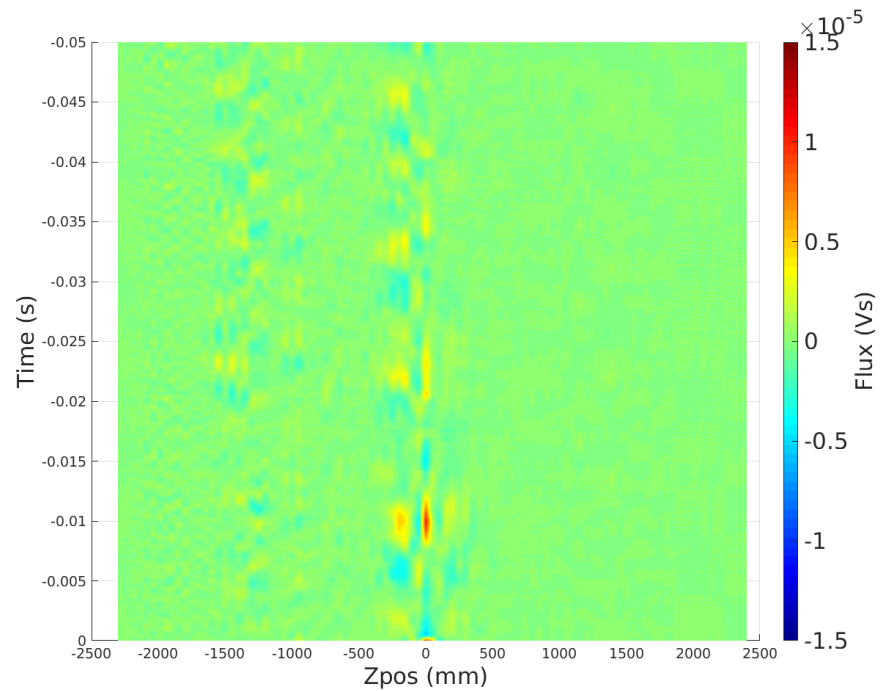

Fig.8. ZQA flux from Quench \#2, showing shift to magnet center.

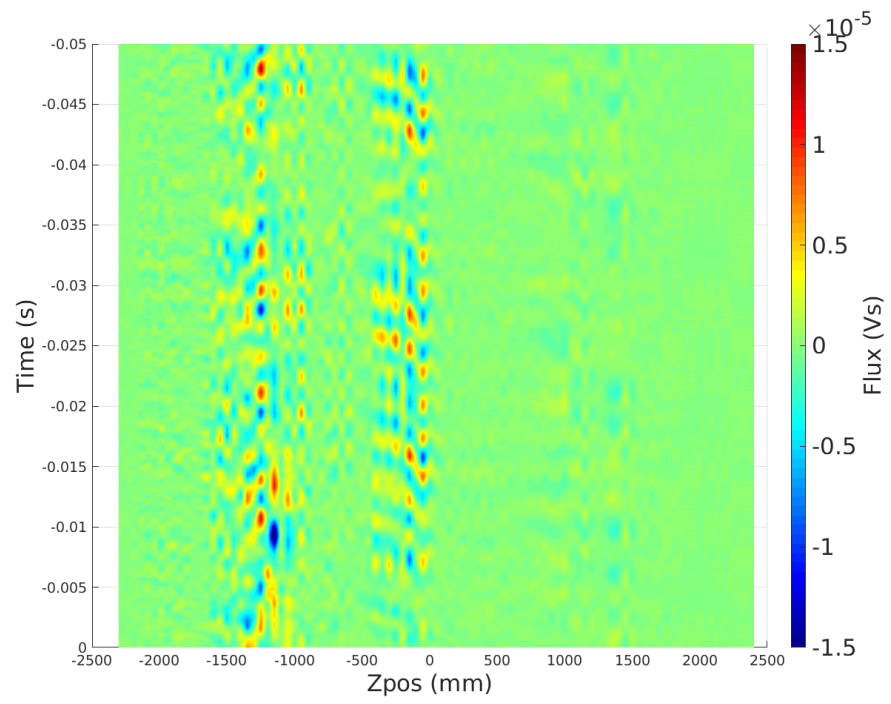

Fig. 9. ZQA cumulative flux for Quench \#3 vs time and axial location. The quench occurs at position $-1150 \mathrm{~mm}$, although there is substantial activity also at center position, as for Quench \#2.

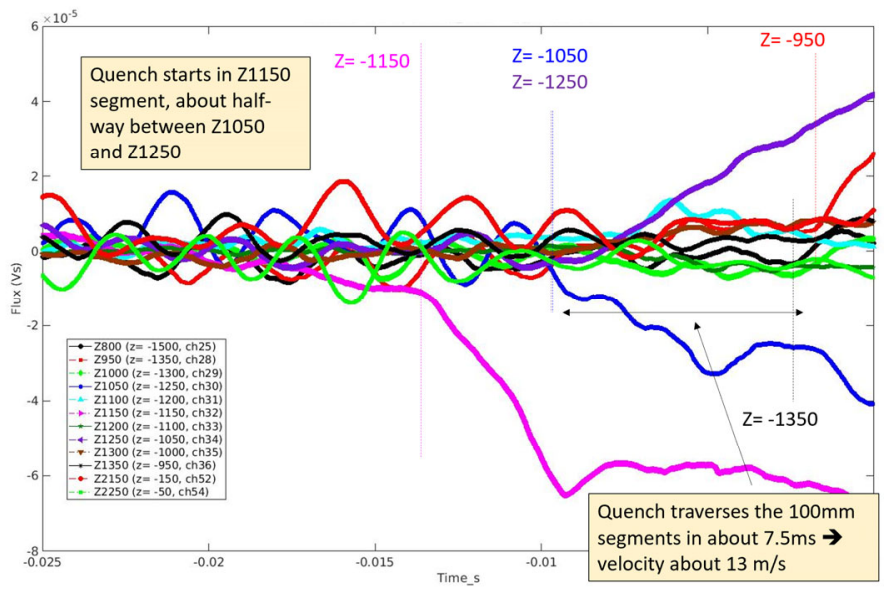

Fig.10. ZQA detection of quench propagation using flux signals in Q\#3.

Theta antennas (TQA) also detect some activity in all quenches. However, analysis has not yielded a good comparison with the partial voltage tap data available in 


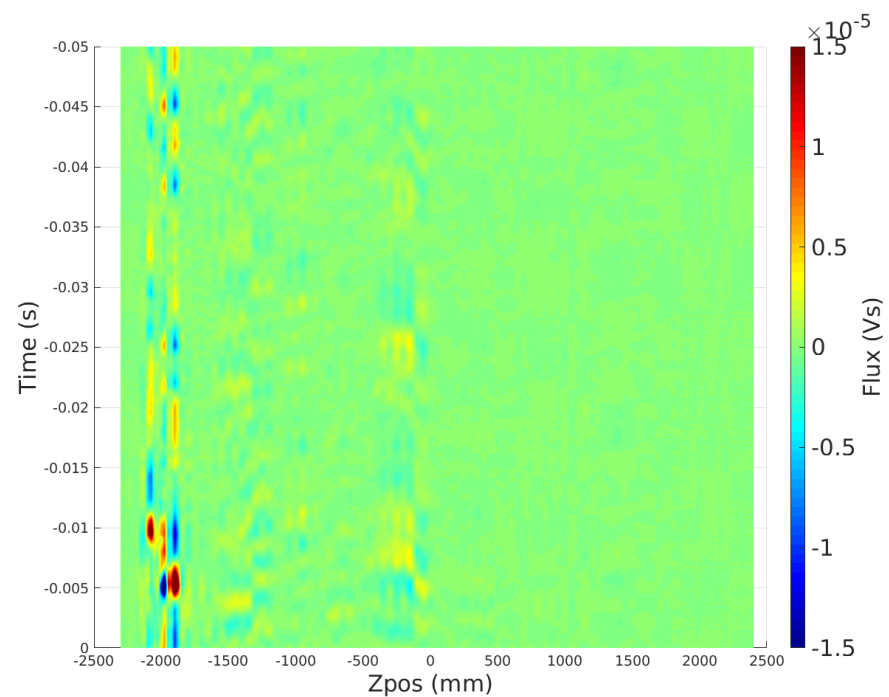

Fig.11. ZQA data showing quench in magnet non-lead end region, Q\#6.

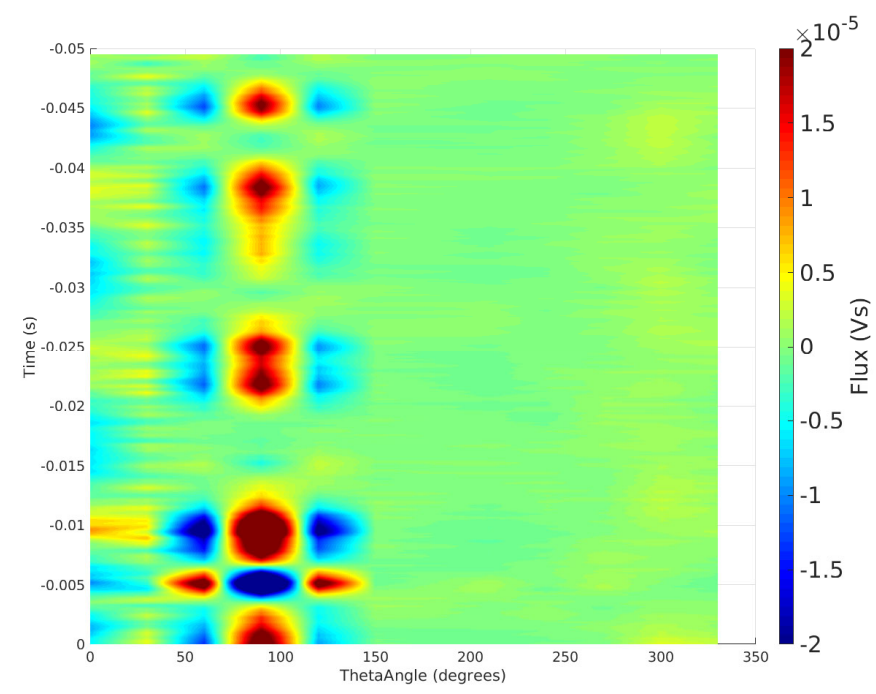

Fig.12. Example of TQA detection of quench angle for Quench \#6

determining angular position. Further data will continue to be taken on subsequent magnets to understand if there are limitations here.

The TQA data require some filtering to help extract the weak, amplified signal. An example is shown in Fig. 12 for Quench \#6; note that with 1000 gain amplification, the TQA signal size of Fig. 12 is on the order of the ZQA signal of Fig. 7. The angular variation at the time slice where the quench occurs $(\sim-10 \mathrm{~ms})$ of the quench of Fig. 12 is similar to the calculated expectation from the simple model of Section III, as shown in Fig. 13.

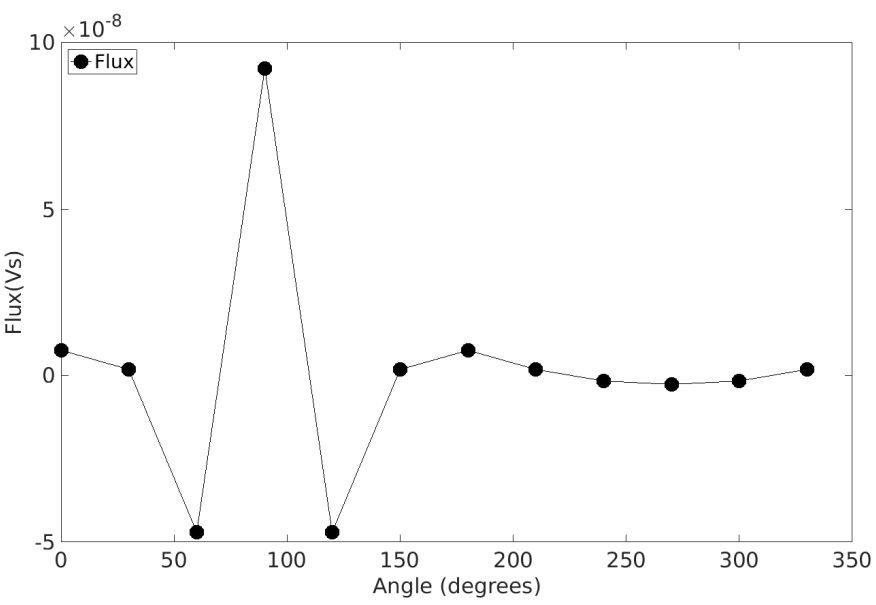

Fig.13. Calculation of expected TQA flux vs angle (for quench at 90 degrees).

Note also that the angular offset of the ZQA can additionally provide some azimuthal location information. The relative sensitivity to a flux event of one angular position of antenna relative to an overlapping one, shifted by 30 degrees, can provide information indicating which one is proportionally closer in angle to the event than the other.

\section{CONCLUSION}

In summary, it appears that the $\mathrm{Z}$ quench antennas have very low noise and high sensitivity - allowing simultaneous measurements of current redistributions as a function of time over the entire magnet - and thus offering a new window on quench development and current redistribution events. The theta antennas also find angular location of quench, but owing to noise and signal size, may be more limited in angular resolution.

\section{ACKNOWLEDGMENT}

The authors would like to thank the expert technical staff at Fermilab and Brookhaven Labs for making realization of this project possible.

\section{REFERENCES}

[1] Ogitsu, T, Devred, A, and Kim, K. Quench antenna for superconducting particle accelerator magnets. United States: N. p., 1993. Web. doi:10.2172/91952.

[2] J. DiMarco et al., "Application of PCB and FDM Technologies to Magnetic Measurement Probe System Development", IEEE Trans. on Appl. Supercond., Vol. 23, Issue 3, 2013, p. 9000505.

[3] J. Muratore, et. al, "Test Results of the First Pre-Series Quadrupole Magnets for the LHC Hi-Lumi Upgrade", proceedings of this conference. 